\title{
Cómo enseñar E/LE para brasileños con el empleo del diccionario en clase: el caso de las marcas de uso
}

\author{
GLAUBER LiMA MOREIRA \\ Universidade Federal do Piauí (UFPI) \\ Universitat Pompeu Fabra (UPF-IULA/INFOLEX) \\ Fundacão CAPES-Ministério da Educação do Brasil \\ EDNA MARIA V. M. ARAúJO \\ Centro de Línguas - IMPARH \\ Universidade Estadual do Ceará (UECE/LETENS)
}

Resumen: El propósito principal del presente trabajo es presentar una secuencia didáctica mediante el uso del diccionario para el desarrollo de la escritura de los estudiantes brasileños de español como lengua extranjera (E/LE), nivel B1 y B2. Hemos considerado las nuevas competencias y prácticas pedagógicas, incluyendo el uso del diccionario en el aula como género textual dirigido a la enseñanza de lenguas. Este artículo está basado en los estudios de Alzola Fariñas (2001), Kleiman (2002, 2013), Araújo (2007), Pontes (2009), Moreira (2009) y Rojo (2013), entre otros. Es importante que el profesor y el estudiante sepan cómo utilizar todas las posibilidades que el diccionario ofrece para el aprendizaje. Trabajamos con las marcas lexicográficas de la lengua (información sobre los aspectos socioculturales de la lengua), como diatécnicas medicina (MED), informática (INF), botánica (BOT), etc. Utilizamos diccionarios monolingües y semibilingües adecuados a los aprendices en la etapa del desarrollo y consolidación de la escritura en E/LE.

Palabras clave: diccionario pedagógico, aprendizaje de la escritura, enseñanza del español, secuencia didáctica.

\begin{abstract}
The main purpose of this work is to present a didactic sequence through the use of dictionary for the development of writing by Brazilian students of Spanish as a foreign language (SFL), levels B1 and B2. We have considered the new pedagogical and practices skills, including the use of dictionary in the classroom as a textual genre aimed at teaching languages. This article is based on the studies of Alzola Fariñas (2001), Kleiman (2002, 2013), Araújo (2007), Pontes (2009), Moreira (2009) and Rojo (2013), among others. It is important that teachers and students know how to use all the possibilities that the dictionary offers for learning. We worked with the lexicographical marks of the language (information on the sociocultural aspects of the language) such as medicine (MED), computing (COMP), botany (BOT), etc. We used suitable monolingual and bilingualized dictionaries for learners in the writing development stage.
\end{abstract}

Keywords: pedagogical dictionary, improving writing, teaching Spanish, didactic Sequence. 


\section{Introducción}

Con la necesidad cada vez mayor de aprender un idioma extranjero, el mercado editorial ofrece, actualmente, diversos materiales educativos como los diccionarios para aprendices (DA), es decir, obras lexicográficas dirigidas para la enseñanza y el aprendizaje tanto de la lengua materna (LM) como de la lengua extranjera (LE). Lo que justifica, en nuestra opinión, el número de estudios que discuten estas obras con el fin de verificar cómo su uso puede ser un elemento didáctico facilitador en este proceso de enseñanza-aprendizaje en el aula.

El diccionario, en general, es considerado un material didáctico ${ }^{1}$ por excelencia, porque presenta diversas informaciones sobre los aspectos léxicos de una lengua, como, por ejemplo, gramaticales, sintácticos, semánticos, fonéticos, etimológicos, culturales y terminológicos, entre otros. Sin embargo, se advierte que esta herramienta se usa poco en el ámbito escolar y que tanto profesores como estudiantes parecen no darse cuenta de su potencial (cfr. Béjoint, 1981), Chi (1988) y Tarp (2006). Es decir, de la importancia y del efecto positivo del uso del diccionario en la adquisición de una lengua extranjera, en particular para la recepción (comprensión y lectura) y producción (habla, escritura y traducción) ${ }^{2}$ del español como lengua extranjera (en adelante E/LE).

De lo expuesto anteriormente, presentamos en este artículo algunas consideraciones acerca de la importancia y de la enseñanza de la escritura, especialmente en lo relativo al E/LE, teniendo en cuenta las nuevas competencias de literacidad y las nuevas prácticas de alfabetización. En este sentido, analizamos la inclusión del uso del diccionario en el aula como género textual (Fuertes-Oliveira, 2010), cuyo recurso está dirigido a la enseñanza de lenguas maternas o extranjeras, en este trabajo el caso del idioma español. Es importante que el profesor y el estudiante sepan cómo utilizar esta herramienta pedagógica lexicográfica, explorando todas las posibilidades que ofrece para la construcción de los conocimientos lingüísticos de una sociedad dada.

Por tanto, presentamos una propuesta didáctica para desarrollar las destrezas de uso del diccionario, además de las actividades para el desarrollo de la escritura y otras alfabetizaciones tocantes a la enseñanza-aprendizaje de E/LE, ya que se trata del empleo de un texto multimodal (Duarte y Pontes, 2013). Dicha propuesta debe cubrir los intereses del alumno de español y, también, llevarlo a desarrollar las nuevas formas para «expresar y representar identidades multifuncionales correspondientes a diferentes estilos de vida» (Rojo, 2013: 15).

\section{Aspectos teóricos}

La actitud del educador, como sujeto consciente de las necesidades e intereses de los estudiantes, así como de las prácticas de literacidad que pueden ser aplicadas en el aula, respetando el potencial de cada uno, podrá marcar la diferencia en el proceso de aprendizaje de estos individuos. También es esencial que el profesor tenga en cuenta la voluntad que el estudiante posea para aprender, sacando ventaja de ello para minimizar las dificultades en el proceso de escritura (Passarelli, 2004) en la lengua española.

\footnotetext{
${ }^{1}$ Véase Jean y Claude Dubois (1971) sobre el carácter didáctico de la obra lexicográfica.

${ }^{2}$ Para más información, consultar los trabajos de (Humblé, 2001, 2006).
} 
Muchos géneros textuales fueron introducidos en la escuela, especialmente en las clases de lenguas maternas y extranjeras, con el objetivo de desarrollar las prácticas de alfabetización de lectura y escritura $\left(P C N S^{3}: 2006 ;\right.$ MCER: 2002), pero solo algunos profesores tratan de llevar a la práctica este tipo de literacidad (Passarelli, 2004) con sus alumnos en el ámbito escolar. Del mismo modo, Kleiman (2000) señala la importancia de la utilización de las «múltiples prácticas de alfabetización de la vida social» como parte esencial de las prácticas experimentadas en la escuela en diferentes niveles de aprendizaje, sugiriendo la realización de «proyectos de alfabetización» de enseñanza. Creemos, de hecho, que esta práctica también se aplica a los cursos de idiomas, los cuales se proponen desarrollar también las destrezas de producción, como la escritura en un código extranjero ( $c f r$. Moreira, 2013: 228). Por tanto, teniendo en cuenta la discusión anterior, proponemos a continuación una secuencia didáctica para el desenvolvimiento de las destrezas en el manejo del diccionario y de la escritura de los estudiantes brasileños de E/LE.

Según Pontes (2009) y Maldonado (2012), es necesario enseñar tanto al profesor como al estudiante a emplear el diccionario para que sus usuarios en potencia tomen el máximo provecho posible de la información que esa obra didáctica ofrece, tales como ortográficas, fonológicas, etimología de la palabra, además de la información gramatical, semántica, pragmática y, también, sobre el grado de especialización en función de los diferentes niveles de la lengua y de los aspectos socioculturales. Por tanto, teniendo en cuenta toda esta información disponible en el diccionario, el consultante de la obra lexicográfica desarrollará por sí solo su autoaprendizaje, la adquisición de vocabulario y la competencia lingüística en el idioma de llegada o lengua meta, en este caso, el idioma español.

\subsection{Breve explicación sobre el género diccionario}

El diccionario es una obra que tiene una arquitectura propia, organizada mayormente en orden alfabético con el fin de facilitar la adecuada consulta de los usuarios de estos recursos didácticos.

Consideramos que el repertorio lexicográfico es un género textual, teniendo en cuenta el estilo, la composición y el contenido presentados por Bakhtin (2003: 266). Según el autor supra «el estilo es inseparable de determinadas unidades temáticas» (ib.), por lo que este género textual permite una ayuda para el usuario en la construcción de significados de un texto y en la producción de otros. Algunos estudiosos de la lexicografía corroboran esta idea, incluyendo a Rey-Debove (1994), a Sobrinho (2000) y a Abad Nebot (2001). Sobrinho (2000: 81), de hecho, sugiere que los diccionarios sean «leídos y no consultados para encontrar respuestas», configurándose como «unidad real de la comunicación discursiva» (Bakhtin, 2003: 268).

Ávila Martín (2000: 16) caracteriza el diccionario como un «objeto», dirigido a la enseñanza, con informaciones que orientan en la «comunicación», escrita y «oral», y que describe los aspectos culturales y lingüísticos de una comunidad lingüística en particular.

Por su parte, Porto Dapena (2000: 35) considera que el diccionario no es fácil de ser caracterizado, ya que se trata de una obra que presenta «una descripción del léxico

\footnotetext{
${ }^{3}$ Parâmetros Curriculares Nacionais.
} 
concebida a modo de fichero, en el que cada ficha viene a ser un artículo donde se estudia una determinada palabra».

Los diccionarios pedagógicos fueron creados para satisfacer las necesidades del aprendiz de la lengua extranjera, que necesita una herramienta que le ayude en la adquisición de vocabulario, en la producción y en la comprensión de textos. Existen dos tipos de diccionarios dirigidos a estas dos últimas funciones, esto es, los diccionarios bilingües y monolingües.

La organización de la obra lexicográfica está constituida por cuatro ejes: megaestructura, macroestructura, medioestructura y microestructura (cfr. Pontes, 2009). El primero es el conjunto de las informaciones desde la introducción hasta los apéndices y anexos. El segundo, la macroestructura, se refiere al conjunto de las entradas que comprenden el diccionario propiamente dicho, por lo general en el orden alfabético. En tercer lugar, la medioestructura, muestra las conexiones entre todas las partes de la obra, es decir, serían los enlaces realizados entre la macro y microestructuras y las otras informaciones de la megaestructura, entre ellas podemos encontrar las remisivas, cuando hay una indicación para referirse a otra palabra o información en el diccionario para más detalles acerca de la palabra de búsqueda. Por último, la microestructura se refiere a la información acerca de cada entrada, formando así el artículo lexicográfico. Según Pontes (2009: 66), los artículos presentados en los diccionarios «consisten en un conjunto de informaciones ordenadas uniformemente, que constituye un programa de información, uniforme y sistemático, organizado en un eje horizontal».

\subsection{Tipos de diccionarios}

En líneas generales, presentamos seguidamente las características generales de algunos tipos de diccionarios. Por ejemplo, el diccionario monolingüe (DM), que, por lo general, ofrece a sus consultantes los artículos a través de una definición sinonímica, información fonética, sintáctica y gramatical sobre la unidad léxica. Tenemos como ejemplo de ello los diccionarios de LM y LE, entre otros. El diccionario bilingüe (DB) es una obra que tiene equivalentes en ambos idiomas, es decir, solo proporciona la traducción (equivalente) en el otro idioma. Por su parte, el diccionario semibilingüe (DS) presenta una definición clara que corresponde a la entrada, en el idioma que se está estudiando (en la LE) y, al final del artículo, incluye el equivalente, esto es, la voz en el idioma del alumno, en otras palabras, el correspondiente en la LM (cfr. Moreira, 2009).

Con respecto a lo anterior, consideramos que es importante que los estudiantes reciban orientación en cuanto a estas informaciones, visto que albergan las características de la obra y los rasgos que pueden diferenciar un diccionario de otro, así como la orientación sobre la estructura y los códigos utilizados por el autor o responsables por su elaboración. Tal conocimiento puede facilitar el manejo y optimizar la consulta del usuario, sobre todo del principiante.

\subsection{Características de los diccionarios escolares utilizados en la secuencia didáctica}

Para este estudio hemos seleccionado los siguientes diccionarios: $i$ ) el semibilingüe Diccionario para la Enseñanza de la Lengua Española para Brasileños (Señas, 2001) y ii) el monolingüe Diccionario de español para extranjeros (2002), para llevar a cabo las 
actividades de la secuencia didáctica. El primero está especialmente dirigido a los estudiantes brasileños, presenta el equivalente y contiene 22.000 entradas, además de informaciones adicionales sobre la regencia y los tipos de complementos verbales, y también aspectos semánticos y marcas de uso. El segundo presenta el vocabulario de español de América Latina y de España, incorpora la voz y la acepción con frecuente uso escrito. Las definiciones son claras y precisas. Existen notas con información ortográfica, morfológica, sintáctica y semántica. El usuario también dispone de una guía de conversación y modelos de conjugación verbal.

En este sentido, creemos que los repertorios lexicográficos elegidos presentan las características ideales de los diccionarios escolares según la etapa de enseñanza de E/LE. Martínez Navarro (2004: 578) presenta dichas características: diseñado a un determinado público, recoge el léxico usual escrito y hablado, presenta definiciones claras, dispone de un reducido número de definidores, marca abundantes ejemplos que reflejan el uso de la palabra, familias de palabras e ilustraciones contextualizadas, además de información complementaria de carácter cultural y enciclopédico.

En función de los marcos teóricos presentados en este estudio, consideramos que el uso efectivo y adecuado de la obra lexicográfica, específicamente el diccionario escolar, será un manual didáctico de investigación constante para nuestro aprendiz brasileño de español como lengua extranjera, dejando de ser simplemente un libro de ayuda puntual para entender solamente el significado de una palabra desconocida. Por tanto, el diccionario se debe concebir como una herramienta de apoyo para el desarrollo de la comunicación y la producción de E/LE.

\section{Aspectos metodológicos}

De lo expuesto hasta ahora, este trabajo tiene el objetivo de desarrollar las destrezas de uso del diccionario escolar en la enseñanza de la escritura en clase de lengua española. En este apartado, presentamos la metodología utilizada para la realización de la secuencia didáctica con marcas de uso en el diccionario. A continuación, exponemos el tema, las marcas de uso seleccionadas y empleadas en la investigación, además de los objetivos de la secuencia didáctica, como también el público meta con el que vamos a trabajar.

\subsection{Selección del tema de la propuesta didáctica}

Como mencionamos anteriormente, el profesor y el estudiante brasileños no utilizan en diccionario adecuadamente, puesto que lo conciben como un mero libro de consulta para resolver diversos problemas en un idioma en particular (cfr. Araújo, 2007). De ahí que sea necesario lograr la alfabetización lexicográfica necesaria, es decir, el usuario, sobre todo el de nivel inicial, necesita saber cómo utilizar el diccionario de manera eficiente en la selección de la información adecuada. Por tanto, vamos a comenzar nuestra propuesta didáctica con actividades diseñadas para comprender la estructura de esta herramienta pedagógica, posteriormente, utilizaremos actividades para la enseñanza de la escrita de E/LE con las marcas lexicográficas que figuran en el repertorio.

Como señalamos en la sección anterior, las marcas lexicográficas son informaciones que encontramos en la microestructura (en los artículos lexicográficos), indican características de uso y son esenciales en el diccionario escolar, ya que «Un extranjero 
que estudia el español como segunda lengua necesita conocer las condiciones de uso de las voces en relación con el contexto en el que éstas actúan» (Salam Zahana, 2012: 105). Como ejemplos de ello podemos citar: (fam.) para familiar, (pop.) para popular, (fig.) para figurado. De ahí que defendamos la importancia de la imprescindible orientación para los lectores de cómo encontrarlas, dado que sus clasificaciones varían según el autor de la obra lexicográfica: las marcas sociolingüísticas, socioprofesionales, socioeconómicas, geográficas, históricas, estilísticas, pragmáticas, de frecuencia, diatécnicas, entre otras.

En este sentido, para este trabajo seleccionamos las siguientes marcas de uso:

-marcas sociolingüísticas: son las informaciones sobre los «niveles lingüísticos y funcionan teniendo en cuenta condiciones de comunicación, y cambian de acuerdo con el medio, las circunstancias, las relaciones sociales existentes entre los individuos», Pontes, (2009: 156) tales como popular (pop.), chulo (chul.), vulgar (vulg.), etc.

-marcas diatécnicas: son aquellas que informan sobre a qué área pertenece la unidad léxica, es decir, si se trata de «la ciencia y la tecnología» (Pontes, 2009), por ejemplo, Medicina (med), Botánica (bot), Informática (inf), etc.

\subsection{Los objetivos de la propuesta didáctica}

1. Desarrollar las habilidades de uso del diccionario monolingüe y semibilingüe para estudiantes brasileños de español como LE, presentando su estructura y los tipos de informaciones que la obra lexicográfica ofrece a su usuario.

2. Fomentar el uso de esta herramienta didáctica en el aula como recurso pedagógico para contribuir a las prácticas de alfabetización relativas a la escritura en $\mathrm{E} / \mathrm{LE}$, a través de actividades sobre las marcas de uso presentadas en los diccionarios disponibles para los estudiantes.

\subsection{Público meta}

La secuencia didáctica fue elaborada para ser empleada por los estudiantes brasileños ${ }^{4}$ de E/LE de los niveles B1 y B2 de los cursos de idiomas. Es importante señalar que estos aprendices ya tienen un conocimiento determinado del código $\mathrm{y}$, por consiguiente, ya están empezando a consolidar la escritura en sus respectivos niveles lingüísticos.

\section{Presentación de la propuesta didáctica}

Esta propuesta didáctica se llevará a cabo en aproximadamente cuatro clases de español, dependiendo del tiempo de que disponga el profesor en el aula con su equipo de estudiantes.

\subsection{Introducción al tema trabajado}

Esta etapa de la propuesta didáctica introduce al alumnado en el tema que se abordará, motivándolo a participar activamente en todas las fases de la actividad. En este tipo de

\footnotetext{
${ }^{4}$ Postulamos que también puede ser utilizada en otros contextos, dependiendo de las necesidades de los aprendientes.
} 
tarea es imprescindible la participación intrínseca del profesor con los estudiantes, puesto que es una actividad de tipo colaborativa, de acuerdo con los siguientes pasos metodológicos:

1. Para iniciar esta actividad el profesor debe asegurarse de que tiene el mayor número de diccionarios en el aula o en la biblioteca del curso.

2. En clase, el profesor comienza una discusión sobre el diccionario a través de cuestiones generales, por medio de las siguientes preguntas, por ejemplo:

a) ¿quién tiene diccionario en casa? En caso afirmativo, ¿cuál es el nombre?

b) ¿quién ya ha manejado un diccionario para ayudar a los estudios? Si la respuesta es afirmativa, ¿cuál usó y qué ha investigado?

c) ¿qué informaciones están disponibles en el diccionario?

3. Después de esta lluvia de ideas, el profesor va a distribuir a los estudiantes, en parejas o en grupos, dependerá de la cantidad de diccionarios disponibles para la actividad, y les pedirá realizar la siguiente tarea:

a) observar la portada y decir el nombre del diccionario y el nombre del autor (si lo hay).

b) observar la portada y decir la cantidad de entradas disponibles en el diccionario y su tipología.

c) abrir el diccionario y comprobar si en las páginas iniciales hay: (1) instrucciones sobre cómo usar el diccionario (una especie de manual); (2) sumario; (3) introducción; y (4) ejercicios con uso del diccionario.

d) examinar, además de las palabras marcadas de A-Z, si existen otras informaciones, tales como: abreviaturas, tablas numéricas, nombres de países/nacionalidades/idioma, listado de verbos, e, incluso, informaciones sobre la historia y la cultura de la lengua en estudio, contenidos estos de gran importancia para un estudiante extranjero ( $c f r$. Atienza Cerezo, 2005).

4. Tras este análisis, es necesario preguntar a los estudiantes lo que es similar y diferente entre los diccionarios analizados. A partir de estas similitudes y diferencias, ellos intentarán definir la estructura (o composición) de este género textual. A continuación, el profesor presentará la estructura del diccionario a través de diapositivas para que los estudiantes puedan comprobar y comparar sus respuestas.

\subsection{Explorando las marcas de uso}

Después de la etapa sobre el conocimiento y sobre el descubrimiento de la estructura del género diccionario, el profesor pasará por la investigación de las marcas de uso que pueden estar presentes en los artículos lexicográficos, de acuerdo con las siguientes estrategias:

1. El primer paso es mostrar que cada lema que el diccionario explica o define se llama artículo lexicográfico (o entrada). De hecho, cada entrada tiene un texto con diversas informaciones, ya sean de orden gramatical, ortográfica, de uso, entre otras. El profesor debe enfatizar que, a pesar de existir una sistematización de la información de los registros, algunos pueden contener más $(+)$ o menos $(-)$ información. Dicho de otra manera, no todos los artículos lexicográficos tienen marcas de uso. 
2. En un segundo momento, es pertinente llamar la atención sobre las informaciones que muestran el uso de la palabra en un contexto determinado, llamadas marcas de uso o marcas lexicográficas. Es importante discutir con los estudiantes cómo se presentan las marcas para cada contexto en que es utilizada la unidad léxica en cuestión. En este trabajo, investigaremos las marcas sociolingüísticas (presentar las marcas seleccionadas) y dialectales (explicarlas) ${ }^{5}$. También debemos mostrar ejemplos de las entradas lexicográficas, extraídas del Diccionario de español para extranjeros, tales como:

a) La voz pachucho (p. 1027) registra dos veces la marca coloquial (col.);

b) En el lema maricón (p. 907), tiene la marca (vulg.).

3. El tercer paso es pedir a los alumnos que seleccionen 10 artículos lexicográficos que contengan marcas de uso, 5 marcas sociolingüísticas y 5 diatécnicas. Enseguida, ellos deben escribir las palabras seleccionadas, las marcas de uso de estas palabras y sus definiciones.

4. Por último, el cuarto paso, cada pareja o grupo hará una exposición, para todos los compañeros del aula, de las palabras elegidas y de las marcas de uso encontradas, incluyendo sus respectivas definiciones. Los alumnos observarán si todas las marcas encontradas son, de hecho, sociolingüísticas o diatécnicas.

\subsection{Producción final de la secuencia didáctica}

Para finalizar la propuesta didáctica los estudiantes producirán un scrapbook con todas las marcas de uso recogidas, colocando figuras ${ }^{6}$ para ilustrar las definiciones. Dicho scrapbook será una especie de glosario ilustrado.

Esperamos que, al final de esta propuesta didáctica, el aprendiz brasileño de E/LE sepa cómo se estructura el diccionario, cuáles son sus características y cómo el referido recurso pedagógico presenta la información lexicográfica. Por consiguiente, el estudiante reconocerá claramente los tipos de informaciones disponibles en esta obra didáctica, como las marcas de uso, las cuales son responsables de las referencias de uso que una palabra puede asumir en un determinado contexto (Gutiérrez Cuadrado, 1996).

Es necesario destacar que el profesor debe planear las actividades de propuestas didácticas con bastante esmero, pero tampoco puede ser muy riguroso en la ejecución de todas las etapas, pues la flexibilidad de las acciones es inherente a cualquier actividad, proporcionando los ajustes necesarios e indispensables (dependiendo del contexto en que está insertado) para tener éxito en el aprendizaje al final de cada tarea educativa.

\section{Conclusiones}

El resultado de este trabajo nos lleva a creer que las actividades realizadas por los estudiantes en la propuesta didáctica los preparan para situaciones reales de la vida cotidiana (Kleiman et al., 2013). Probablemente, los cursos de idiomas no tienen el conocimiento y la preparación necesarios de cómo se podría utilizar el diccionario como soporte pedagógico facilitador y motivador en las clases de lenguas extranjeras (Araújo, 2007), especialmente en la enseñanza de la lengua española, enfoque de la presente

\footnotetext{
${ }^{5}$ Véase Pontes (2009), Welker (2004) y Alzola Fariña (2001).

${ }^{6}$ El profesor ofrecerá revistas y pedirá a los alumnos que también lleven al aula.
} 
propuesta didáctica, a través de las marcas lexicográficas sociolingüísticas y diatécnicas. Consideramos que estos registros facilitan su comprensión y utilización en los contextos específicos que pueden ser utilizados en la lengua de estudio que contribuyen eficazmente a la enseñanza del código lingüístico.

Es casi seguro que la mayoría de los alumnos tienen al menos un diccionario en casa (creencia de que un diccionario sirve para toda la vida), adquirido, muchas veces, por la propia incentiva del profesor. Incluso los cursos de idiomas, a veces, tienen diccionarios en su biblioteca. Por tanto, es necesario explorar este género en el aula, tanto para informar a los estudiantes sobre su organización y lo que ofrece a los consultantes, como para enseñarles a buscar las informaciones necesarias para resolver los problemas lingüísticos y a emplearlo como auxilio para el desarrollo de la escritura en E/LE.

También concluimos que los profesores de LE debemos diversificar las prácticas pedagógicas para que los estudiantes se sientan motivados e interesados en aprender una LE y a participar de las prácticas desarrolladas en la clase de lenguas. Como afirman Kleiman et al. (2013: 81) «Cuando los estudiantes están involucrados en proyectos de alfabetización que les interesan, ellos objetivan actuar sobre el mundo a través de actividades de lenguaje [...] para lograr sus propósitos»».

Por último, consideramos que el diccionario escolar está poco o inadecuadamente insertado en el aula para el desarrollo de la enseñanza y del aprendizaje de una lengua, por lo que los contenidos lingüísticos, enciclopédicos y culturales que la obra dispone no están siendo plenamente explotados. Por tanto, creemos que, como género textual, el diccionario escolar es una herramienta que pretende contribuir a la expansión del conocimiento de los alumnos, lingüística y culturalmente hablando, tanto en los aspectos formales de la lengua como en las prácticas socioculturales de la comunidad nativa de esta lengua y debe ser ampliamente utilizado como recurso didáctico.

\section{Bibliografía}

AbAd NeBot, F. (2001). «Cuestiones de lexicología y Lexicografia». Madrid: UNED ediciones.

Alzola FariÑas, L. F. (2001). «Las presentaciones de los diccionarios escolares. Breve historia de un elemento didáctico olvidado por las editoriales, los profesores y los usuarios». Glosas Didácticas. n. 6. Disponible en http://sedll.org/doces/publicaciones/glosas/n6/alzola.html. [Consulta: 05/04/2017].

ArAúJo, E. M. V. M. (2005). «O dicionário para aprendizes em sala de aula: uma ferramenta de ensino e aprendizagem». Memoria de Máster (Programa de Pós-graduação em Linguística Aplicada), Universidade Estadual do Ceará, Fortaleza - CE.

AraÚJo, E. M. V. M. (2007). «O dicionário Monolíngue para aprendizes em sala de aula: uma ferramenta de ensino/aprendizagem». Horizontes de Lingüística Aplicada, 6(2), 254272.

Atienza Cerezo, E. (2005). «El tratamiento de la cultura en los diccionarios de aprendizaje», Revista electrónica de didáctica del español lengua extranjera (redELE); vol. 2 (5), 1-8, Madrid. 
Ávila Martín, M. del C. (2000). «El diccionario en el aula». Granada: Editorial Universidade de Granada, Granada.

Bakhtin, M. (2003). «Estética da criação verbal». São Paulo, Martins Fontes.

BÉJOINT, H. (1981). «The foreign student's use of monolingual English dictionaries: a study of language needs and reference skills». Applied Linguistics, v. II, 3, 207-222.

CHI, M. L. A. (1998). «Teaching dictionary skills in the classroom». En EURALEX' 98 Proceedings. Belgium, 565-578.

CONSEJO DE EuRopa. (2002). «Marco Común Europeo de Referencia para las lenguas: Aprendizaje, Enseñanza, Evaluación». Madrid: MECD-Instituto Cervantes. Recuperado el 02/03/2017 de http://cvc.cervantes.es/ensenanza/biblioteca_ele/marco

DuARTE, E. y PonTES, A. L. (2011). «O componente medioestrutural do minidicionário escolar Caldas Aulete». Revista Entrepalavras, v.1, n. 1, 48-57.

Fuertes-Oliveira, P. A. (2010). Specialised dictionaries for learners. Berlín: De Gruyter.

GUTIÉRREZ CUADRADO, J. (1996): «Las marcas en los diccionarios para extranjeros». En Diez de Revenga, P. y Jiménez Cano, J. M. (eds.). Estudios de Sociolingüística. Sincronía $y$ diacronía (pp. 95-106). Murcia: DM.

HERnÁNDEZ, H. (2000) «El diccionario en la enseñanza de E.L.E (Diccionarios de español para extranjeros)». En Zorraquinho, M. A. M y Pelegrín, C. D. (Org.)¿Qué español enseñar? Norma y variación lingüisticas en la enseñanza del español a extranjeros (pp. 92-102). Zaragoza: Universidad de Zaragoza.

Kleiman, A. B. (2000). «O processo de aculturação pela escrita: ensino da forma ou aprendizagem da função?». En Kleiman, A. y B. Signorini, I. (Org.) O ensino e a formação do professor. Alfabetização de Jovens e Adultos (pp. 223-243) Porto Alegre: Artmed.

Kleiman, A. B.; Ceniceros, R. C. y Tinoco, G. A. (2013). «Projetos de Letramento no ensino médio». En Buzen, C. y Mendonça, M. (org.). Múltiplas linguagens para o ensino médio.(pp. 69-83). São Paulo: Parábola.

Humblé, P. (2001). Dictionaries and language learners. Frankfurt am Main: Haag und Herchen.

Humblé, P. (2006). «Melhor do que muitos pensam. Quatro dicionários bilíngües português-inglês de uso escolar». En Xatara, C. y Humblé, P. Cadernos de Tradução: Tradução e Lexicografia pedagógica (pp. 253-274). Universidade Federal de Santa Catarina. Centro de Comunicação e expressão. Florianópolis: Pós-Graduação em Estudos da Tradução. n. XVIII.

MALDONAdO, C. (2012). «Los diccionarios en el mundo ELE: ayer, hoy y mañana: una reflexión desde la propia experiencia», Revista Internacional de Lenguas Extranjeras, 1, 151-179.

Martínez NAVArro, M. del R. (2004). «El diccionario de L2 que "deseamos"». En Castillo Carballo, M. A. et al (org.). Las gramáticas y los diccionarios en la enseñanza del español como segunda lengua: deseo y realidad. Sevilla: Universidad de Sevilla. 
MoreIRA, G. L. (2007). «O uso do dicionário monolingüe na sala de aula: uma ferramenta para a compreensão leitora em língua espanhola por alunos avançados de espanhol/LE. Memoria de Máster (Programa de Mestrado Acadêmico em Linguística Aplicada). Fortaleza: Universidade Estadual do Ceará.

MoreIRA, G. L. et al. (org.). (2013). Reflexões e ações no ensino e aprendizagem de Espanhol/LE. Fortaleza: EdUECE.

Passareli, L. G. (2004). «Ensinando a escrita: o processual e o lúdico». São Paulo: Cortez, 79-101.

Pontes, A. L. (2009). Dicionário para uso escolar: o que é, como se lê. Fortaleza: EdUECE.

Porto Dapena, J. A. P. (2002). Manual de técnica lexicográfica. Madrid: Ibérica Grafic, Arco/Libro, S.A.

REY-DeBove, J. (1971). Étude linguistique et semiotique dês diccionnaires françaises contemporaines. Paris: Hachette.

Rojo, R. (2013). «Gêneros discursivos do Círculo de Bakhtin e multiletramentos». En Rojo, R. (Org.). Escola conectada: os multiletramentos e as TICs (pp. 13-36). São Paulo: Parábola.

Salam ZahanA, A. M. A. El. (2012). «Sobre la necesidad de marcar aspectos pragmáticos en el Diccionario bilingüe (español-árabe) de F. Corriente». En Nomdedeu Rull, A. et al. (ed.). Avances de lexicográfia hispánica (II). Tarragona: Publicacions $\mathrm{URV}, 1 .^{\mathrm{a}}$ ed., 2 vols.

SECRETARIA de EDUCAÇÃo BÁSICA DE BRASIL. (2006). Orientações curriculares para o ensino médio: linguagens, códigos e suas tecnologias. Brasília: Ministério da Educação. Recuperado el 08/05/2016 de http://www.mec.gov.br.

SEÑAS. (2001). Diccionario para la enseñanza de la lengua española para brasileños. Universidad de Alcalá de Henares. $2^{\mathrm{a}}$ ed. São Paulo: Martins Fontes.

VIEIRA (2013). «Letramento digital: o uso de tecnologías da informação e da informação e da comunicação no ensino da leitura». Anais do SILEL. V. 3, n. 1, Uberlândia: UDUFU.

SoBRINHO, J. C. (2000). «Uso do dicionário: configurando estratégias de aprendizagem do vocabulário». En Leffa, V. J. As palavras e sua companhia: o léxico na aprendizagem. Pelotas: ALAB.

TARP, S. (2006). «Lexicografía de aprendizaje». Cadernos de Tradução, v. 2, n. 18, $295-$ 317. Recuperado el 20/04/2017 de: https://periodicos.ufsc.br/index.php/traducao/article/view/6974

VV.AA. (2002). Diccionario de español para extranjeros. Madrid: Ediciones SM.

XAVIER, A. C. (2011). «Letramento digital: impactos das tecnologias na aprendizagem da Geração Y». Calidoscópio, v. 9, n. 1, 3-14. 